\title{
Measurement of the direct particle transport through stochastic media using neutron resonance transmission analysis
}

\author{
Bjorn Becker ${ }^{1, a}$, Stefan Kopecky ${ }^{1}$, Hideo Harada $^{2}$, and Peter Schillebeeckx ${ }^{1, b}$ \\ 1 European Commission, Joint Research Centre, Institute for Reference Materials and Measurements, Retieseweg 111, \\ B-2440 Geel, Belgium \\ 2 Japan Atomic Energy Agency, Tokai-mura, Ibaraki, 319-1195, Japan
}

Received: 22 November 2013 / Revised: 16 January 2014

Published online: 15 April 2014

(c) The Author(s) 2014. This article is published with open access at Springerlink.com

\begin{abstract}
A measurement of the uncollided neutron flux passing through a sample containing a stochastic mixture of tungsten and sulfur grains has been performed using neutron resonance transmission analysis in the $3-200 \mathrm{eV}$ energy region. The impact of the heterogeneous characteristic of the sample is shown, based on a comparison of the measurement with a calculated transmission spectrum of a homogeneous sample, which was verified by a measurement with a homogeneous metallic disc. By using a single strong resonance of tungsten, the particle self-shielding factor between $0.2-0.9$ was directly measured. The experimental data have been compared with model calculations using the Markovian Levermore-Pomraning model. The measured transmission has been used to determine the effective characteristic chord length and volume fraction of the tungsten grains within the sample.
\end{abstract}

\section{Introduction}

When particles (e.g. neutrons, photons) travel through a stochastic mixture, the attenuation of the uncollided particle flux can differ significantly from the attenuation in a homogenized medium. This occurs predominantly if the characteristic grain size length is larger than the mean free path in the grains. The effect is commonly known as particle self-shielding [1], where particle refers to the powder grains. In this contribution, this self-shielding effect of tungsten grains in a mixture with sulfur grains is investigated by neutron resonance transmission analysis (NRTA). A transmission measurement using neutrons is a well-established method to determine neutron-induced total cross sections [2]. By applying the time-of-flight (TOF) technique characteristic resonance structures of nuclei can be revealed. In case the cross section is accurately known, NRTA can be used as a non-destructive method to measure the elemental and isotopic sample composition [3].

The necessity to include the stochastic nature of the media in the calculation of the particle transport occurs in various scientific fields. For example, in radiation protection, the effectiveness of shielding materials such as concrete can depend on the homogeneity of the material, in particular, if strong absorber grains are added to the shielding. The effectiveness of the shielding depends then strongly on the size and heterogeneous distribution of the added grains [1, 4]. In nuclear engineering, neutron transport calculations need to account for stochastic mixtures such as the two-fluid turbulent mixture of liquid water and vapor in a boiling water reactor [5] or the randomly dispersed burnable poison grains in nuclear fuel elements [6]. In climatology, the calculation of the radiation transfer through a partially cloudy atmosphere as component of a general circulation model has to include a model for the stochastic mixture of clouds and clear sky [7-9]. In astrophysics, calculations of the interstellar photon transport may need to take into account the clumpiness of molecular clouds $[10,11]$.

Various analytical models have been developed over the years to calculate the transmission of particles through stochastic media based on different underlying stochastic assumptions and approximations $[1,4,5,12-17]$. We refer the reader to the original publications for specific details of the different models. In this contribution we focus on the Levermore-Pomraning (LP) model [15] since it is widely applied in various fields.

In the past, the transport of particles through a stochastic mixture has been studied experimentally. At the Oak Ridge National Laboratory, the thermal neutron flux of the ORNL Graphite Reactor was used to study the

\footnotetext{
a e-mail: bjorn.becker@ec . europa.eu

b e-mail: peter.schillebeeckx@ec. europa.eu
} 
transmission of neutrons through a stochastic mixture of boron-carbide and aluminum [14]. Doub [1] studied the particle self-shielding of boron-carbite spheres by measurements with a crystal spectrometer using low-energy neutrons $(<2 \mathrm{eV})$. The data were limited to a minimum particle self-shielding factor of 0.86 . Rosen et al. [18] and Keiter et al. [19] studied the photon transport through inhomogeneous plasmas and foams, respectively, using laser beams. The experiment presented in this study is in complement to the previous experimental results by analyzing the neutron transmission through a stochastic mixture using resonance structured profiles to cover a large range of particle selfshielding. In addition, the resonance structured profile of the transmission was used to deduce characteristics of the heterogeneous sample, namely the effective characteristic length and volume fraction of the tungsten grains. For this it was assumed that the sample can be described by a stationary, binary Markovian mixture and that only uncollided neutrons which passed the sample are detected.

In sect. 2 the LP model of particle transport through a stochastic medium is briefly summarized. In sect. 3 both the experimental setup of the measurement and the data reduction are described. Results are given and discussed in sect. 4 .

\section{LP model of the particle transport through stochastic media}

Levermore et al. [15] developed a model for the particle transport through a stationary Markovian statistical binary mixture. This model is often referred to as the LP model [20]. The LP model has been applied, for example, in climatology to calculate the radiation through cloud fields [8], in inertial confined fusion and to calculate the radiation transfer through a two-fluid turbulent mixture of liquid water and vapor [15].

Within this model the line segments in a particular component along a trajectory of a particle have an exponential decaying length probability distribution. The uncollided, ensemble averaged, monoenergetic particle flux $\Psi$ at position $x$ is given by [15]

$$
\Psi(x)=\Psi_{0}\left[\frac{r_{+}-\tilde{\Sigma}}{r_{+}-r_{-}} e^{-r_{+} x}+\frac{\tilde{\Sigma}-r_{-}}{r_{+}-r_{-}} e^{-r_{-} x}\right],
$$

where $\Psi_{0}$ is the initial flux at position zero. The decay constants $r_{ \pm}$are given by

$$
2 r_{ \pm}=[\langle\Sigma\rangle+\tilde{\Sigma}] \pm\left[(\langle\Sigma\rangle-\tilde{\Sigma})^{2}+4 \beta\right]^{1 / 2}
$$

with

$$
\begin{aligned}
\langle\Sigma\rangle & =p_{\alpha} \Sigma_{t}^{\alpha}+p_{\beta} \Sigma_{t}^{\beta} \\
\tilde{\Sigma} & =p_{\beta} \Sigma_{t}^{\alpha}+p_{\alpha} \Sigma_{t}^{\beta}+\lambda_{\alpha}^{-1}+\lambda_{\beta}^{-1} \\
\beta & =\left(\Sigma_{t}^{\alpha}-\Sigma_{t}^{\beta}\right)^{2} p_{\alpha} p_{\beta} .
\end{aligned}
$$

The two different components of the binary mixture are denoted by $\alpha$ and $\beta$. $\Sigma_{t}^{i}$ and $\lambda_{i}$ are the total, Doppler broadened, macroscopic cross section and the mean chord length of component $i$, respectively. The volume fraction $p_{i}$ is given by $p_{i}=\lambda_{i} /\left(\lambda_{\alpha}+\lambda_{\beta}\right)$.

The transmission $T_{\mathrm{LP}}$ of a monoenergetic particle beam through a sample with a thickness $t$ is simply

$$
T_{\mathrm{LP}}=\frac{\Psi(t)}{\Psi_{0}}
$$

On the other hand, the transmission $T_{\text {hom. }}$ of particles through a homogeneous sample is given by the Beer-Lambert law consisting of only one decaying exponential,

$$
T_{\text {hom. }}=e^{-\left(p_{\alpha} \Sigma_{t}^{\alpha}+p_{\beta} \Sigma_{t}^{\beta}\right) t}=e^{-\langle\Sigma\rangle t} .
$$

The microscopic and macroscopic cross section of component $i$ are then related by: $p_{i} t \Sigma_{t}^{i}=n_{i} \sigma_{i}^{t}$ with the areal density denoted by $n_{i}$.

The particle self-shielding factor $f$ is used as a correction factor of the homogenized cross section $\langle\Sigma\rangle$ to take into account heterogeneity effects. The transmission of a monoenergetic particle beam through a heterogeneous sample $T_{\text {het. }}$ is then [1]

$$
T_{\text {het. }}=e^{-f\langle\Sigma\rangle t} \text {. }
$$

Using the definition of the transmission within the LP model (eq. (6)) leads to

$$
f_{\mathrm{LP}}=\frac{-\ln \left(T_{\mathrm{LP}}\right)}{\langle\Sigma\rangle t}=\frac{\ln \left(T_{\mathrm{LP}}\right)}{\ln \left(T_{\text {hom. }}\right)} .
$$




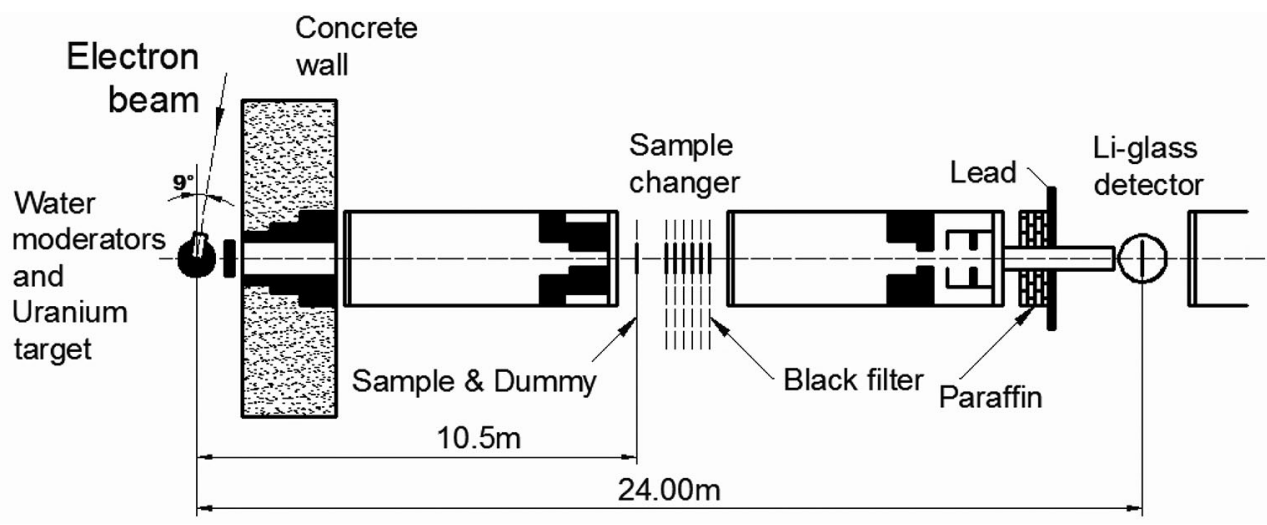

Fig. 1. A schematic representation of the transmission set-up.

The LP model (eqs. (1)-(6)) has recently been implemented directly into the resonance shape analysis code REFIT [21] for the analysis of transmission data. Details of this implementation can be found in ref. [22]. Using numerical derivatives REFIT can fit all model parameters in the same manner as it fits resonance parameters or areal densities.

\section{Experimental conditions and data analysis}

The self-shielding was investigated using neutrons as interrogation particles in a transmission experiment. The measurements were carried out at the GELINA (GEel LINear Accelerator) TOF facility of the EC-JRC-IRMM [23]. GELINA is a multi-user, multi-purpose facility built for high-resolution neutron spectroscopy with a white neutron source with neutron energies ranging from $10 \mathrm{meV}$ to $20 \mathrm{MeV}$. It uses a pulsed linear electron accelerator which provides a beam with a maximum energy of $150 \mathrm{MeV}$, a repetition rate between 50 and $800 \mathrm{~Hz}$ and a peak current of $12 \mathrm{~A}$. A magnet is used to compress the pulsed electron beam to about 1 ns before it impinges on a mercury-cooled rotating uranium target and Bremsstrahlung is generated. Neutrons are produced by $(n, \gamma)$ and $(n, f)$ reactions. To produce a white neutron spectrum ranging from thermal energy up to a few $\mathrm{MeV}$, two $4 \mathrm{~cm}$ thick beryllium containers filled with water are placed beneath and above the target as moderators. By shielding either the target or the moderator, the direct or moderated spectrum can be used. For the measurements discussed in this contribution the moderated neutron spectrum was used.

\subsection{Experimental Setup}

Flight path 2 of GELINA was used for the measurement. This flight path forms an angle of $9^{\circ}$ with the normal of the moderator surface viewing the flight path. A schematic view of the full setup is shown in fig. 1.

During the measurement the accelerator was operated at $800 \mathrm{~Hz}$ with an average electron current of $70 \mu \mathrm{A}$. The total neutron intensity was monitored by two $\mathrm{BF}_{3}$ proportional counters located in the concrete ceiling of the target hall. The neutron beam was collimated in the concrete shielding of the target hall as well as in the 50 cm diameter flight tubes. At the sample position the beam had a diameter of $2 \mathrm{~cm}$. The sample was placed in an automatic sample changer at $10.5 \mathrm{~m}$ distance from the neutron producing target, allowing for an alternated sample-in and sample-out measurement. Close to the sample position a ${ }^{10} \mathrm{~B}$ overlap filter, with an areal density of $0.008 \mathrm{at} / \mathrm{b}$, was placed to absorb slow neutrons from a previous burst. The measurement was performed with permanent $\mathrm{S}$ and Na black resonance filters. Additional measurements with $\mathrm{Co}, \mathrm{W}$ and Ag filters were performed to determine the time dependence of the background.

After sample and filters the beam was again collimated and impinged on a NE905 Li-glass scintillator enriched $95 \%$ in ${ }^{6} \mathrm{Li}$, which was placed at a $24 \mathrm{~m}$ flight path. The neutron detector had a diameter of $110.0 \mathrm{~mm}$ and a thickness of $12.7 \mathrm{~mm}$. The detector was viewed by a EMI9823-QKB photomultiplier (PMT). The anode pulse of the PMT was fed into a constant fraction discriminator to create a fast logic time signal, providing the time of arrival of the neutron, and into a spectroscopic amplifier to determine the energy deposited in the detector. The TOF of a neutron was determined using the time difference between the start signal $T_{0}$, given at each electron burst, and the stop signal $T_{s}$ from the scintillator. It was measured with a multi-hit fast time coder with a 0.5 ns resolution, developed at the EC-JRC-IRMM [24]. The TOF was then calculated using

$$
T O F=\left(T_{s}-T_{0}\right)+t_{0},
$$

where the offset $t_{0}$ was determined using the $\gamma$-flash. Both flight path stations with sample and detector were climatized to keep a constant room temperature of $22^{\circ} \mathrm{C}$. 


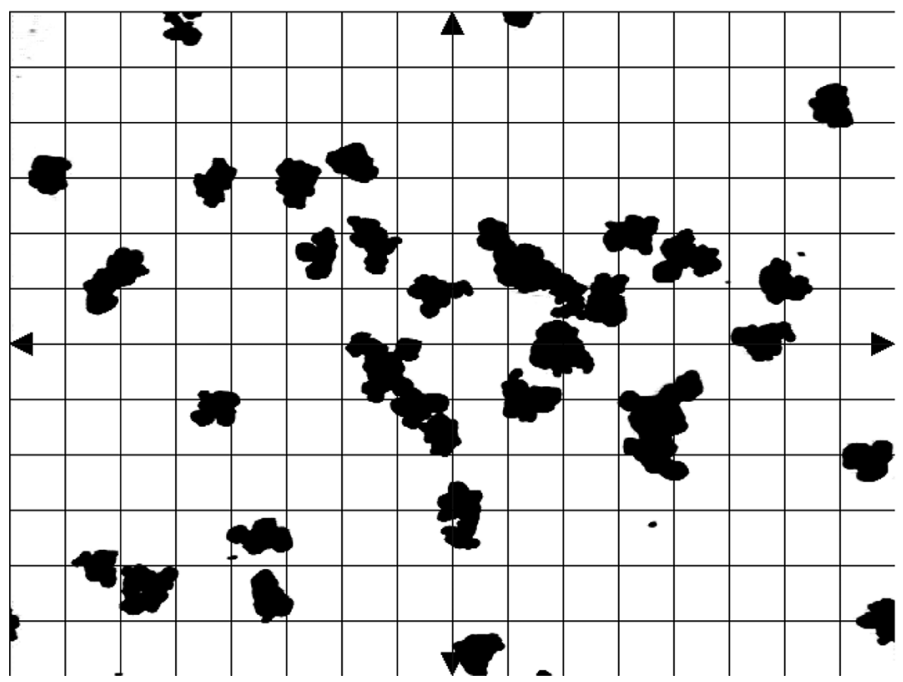

Fig. 2. Photomicrograph of tungsten grains (grid mesh size of $150 \mu \mathrm{m}$ ) before mixing with sulfur powder in the sample preparation process.

The used powder sample was prepared by the target preparation group of the EC-JRC-IRMM [25]. The sample was made by mixing $14.36 \pm 0.10 \mathrm{~g}$ tungsten powder with $3.480 \pm 0.025 \mathrm{~g}$ sulfur powder with nominal grain sizes of 50-250 $\mu \mathrm{m}$ and $44 \mu \mathrm{m}$, respectively. Figure 2 shows a photomicrograph of the used tungsten grains. The tungsten and sulfur powder were mixed for 2 hours in a Teflon container. The mixture was then filled into an aluminum canning with a diameter of $80.01 \pm 0.01 \mathrm{~mm}$, slightly pressed to a thickness of $1.02 \pm 0.07 \mathrm{~mm}$ and closed with an aluminum lid.

For a homogeneous sample the average areal density of the sample is usually determined from the sample mass and area. However, an X-ray radiograph of the powder sample revealed that the sample was not filled completely with the mixed powder. Due to the vertical positioning of the sample in the neutron beam a void was formed at the top of the sample. The sample was positioned with the neutron beam focusing on its lower part. The effective tungsten areal density was then obtained by a fit to small resonances as will be discussed in sect. 4 . Due to the relatively large collimation compared to the grain sizes the neutron beam passes through a significant number of different mixing realizations and an ensemble averaged signal is measured.

For reference, a homogeneous tungsten metal disc was used as well. The sample had a diameter of about $80 \mathrm{~mm}$ and a thickness of about $0.15 \mathrm{~mm}$. The sample area and mass were measured to be $4991.815 \pm 0.134 \mathrm{~mm}^{2}$ and $14.291 \pm 0.001 \mathrm{~g}$. The resulting areal density was $(9.3781 \pm 0.0007) \times 10^{-4}$ at $/ \mathrm{b}$.

\subsection{Data reduction}

The transmission $T_{\text {exp }}$ was obtained from the ratio of a sample-in measurement $C_{\text {in }}$ and a sample-out measurement $C_{\text {out }}$, both corrected for their background contributions $B_{\text {in }}$ and $B_{\text {out }}$, respectively,

$$
T_{\text {exp }}=N \frac{C_{\text {in }}-k B_{\text {in }}}{C_{\text {out }}-k B_{\text {out }}}
$$

The TOF spectra $\left(C_{\text {in }}, B_{\text {in }}, C_{\text {out }}\right.$ and $\left.B_{\text {out }}\right)$ in eq. (11) were corrected for losses due to the dead time in the detector and electronics chain. All spectra were normalized to the same TOF bin width and neutron beam intensity. The latter was deduced from the response of the $\mathrm{BF}_{3}$ neutron monitors. For the mixed powder sample, the sample-out measurement consisted of a measurement using a dummy aluminum canning. The factors $N$ and $k$ were introduced to account for the uncertainty due to the normalization of the beam intensity and due to the used background model, respectively. To avoid systematic effects due to slow variations of both the beam intensity and detector efficiency as a function of time, data were taken by alternating sample-in and sample-out measurements in cycles of about $900 \mathrm{~s}$ each. Applying such a procedure, the factor $N$ is about $N=1.000 \pm 0.0025$ [2].

To determine the background the black resonance technique was applied [2]. The background as a function of TOF was parameterized by an analytical expression consisting of a constant and three exponentials,

$$
B(t)=b_{0}+b_{1} e^{-\lambda_{1} t}+b_{2} e^{-\lambda_{2} t}+b_{3} e^{-\lambda_{3}\left(t+t_{0}\right)} .
$$

The first exponential is due to the detection of $2.2 \mathrm{MeV} \gamma$-rays resulting from neutron capture in hydrogen present in the moderator. The second exponential originates predominantly from neutrons scattered inside the detector station. 

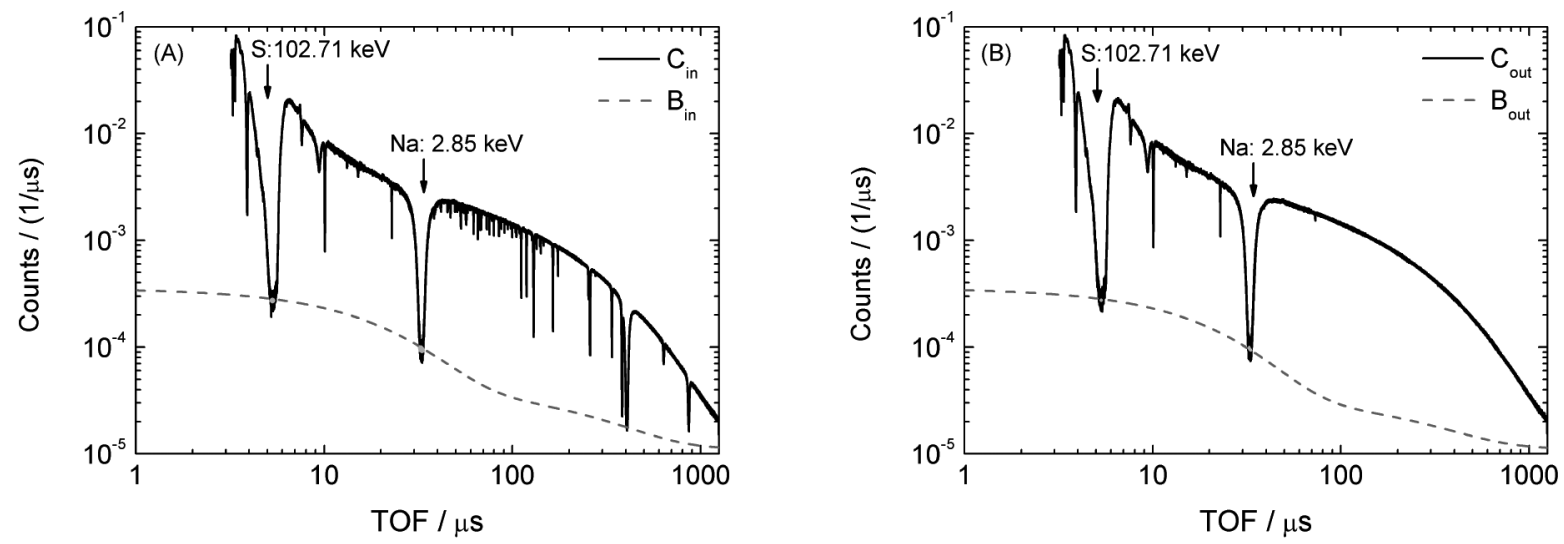

Fig. 3. Sample-in (A) and sample-out (B) TOF spectra together with the corresponding background contribution using the powder sample and dummy, respectively.

The third exponential is due to overlap neutrons from a previous cycle. The parameters $b_{3}$ and $\lambda_{3}$ were determined by fitting the transmission above $1 \mathrm{~ms}$. The time shift $t_{0}$ is the inverse of the used accelerator frequency. The remaining free parameters in the analytical expression $\left(b_{0}, b_{1}, b_{2}, \lambda_{1}, \lambda_{2}\right)$ were determined by a least square fit to saturated resonance dips observed in the TOF spectra resulting from measurements with black resonance filters. The time dependence of the background was derived from dedicated measurements with $\mathrm{S}$, Na, Co, W and Ag black resonance filters in the beam. During the regular sample-in and sample-out runs S and Na fixed black resonance filters were kept in the beam to account for the dependence of the background level on the presence of the sample [2]. The dead time corrected sample-in and sample-out TOF spectra together with the background contributions are shown in figs. 3(A) and (B), respectively. Due to the measurements with the additional black resonance filters, the background uncertainty is less than $3 \%$ which was introduced in eq. (11) using $k=1.00 \pm 0.03$.

The zero point of the time scale was deduced from the position of the $\gamma$-ray flash. The conversion from TOF to energy is also related to the response function of the TOF spectrometer, which is a convolution of different components such as the finite duration of the accelerator burst, the time resolution of the detector and electronics and the neutron transport in the neutron producing target/moderator and the detector [2]. For this measurement, the response is dominated by the neutron transport in the target/moderator assembly which has been simulated numerically [26]. This response is represented by a distribution of an equivalent distance [2]. An average flight path was taken to represent the data in energy.

The data processing package AGS (Analysis of Geel Spectra) was used to derive the transmission spectrum together with the full covariance information [27].

\section{Results}

\subsection{Transmission spectrum}

The experimental transmission spectrum was deduced using eq. (11) and compared to calculations using the resonance shape analysis code REFIT [21]. In the calculation of the transmission $\tilde{T}$ the REFIT code includes the response function of the spectrometer. The REFIT calculations start from tabulated resonance parameters and also include the Doppler broadening of the cross section.

In order to be less dependent on the quality of the nuclear data, the resonance parameters of tungsten were adjusted with REFIT based on a transmission measurement using the homogeneous metal disc as reference sample. The JEFF3.2T evaluation [28] was used as a start file. Figure 4 compares for the homogeneous metal disc the experimental and calculated transmission spectra. The lower part of the figure shows the residual $R$ of the calculation which was determined using

$$
R=\frac{T_{\exp }-\tilde{T}}{u_{T_{\exp }}},
$$

where $\tilde{T}$ is the calculated transmission, $T_{\exp }$. is the measured transmission and $u_{T_{\exp }}$ is the uncorrelated uncertainty of the measurement due to counting statistics. The flat residual, together with the Chi-square per degree of freedom $\chi^{2} / \nu=1.0$, indicate the good quality of the used resonance parameters.

Since the cross section of sulfur is constant and only about $1 \mathrm{~b}$ in the considered energy range, the average sulfur content of $n_{S}=1.300 \times 10^{-3}$ at/b, determined from the total sulfur mass and the sample area, was used in the calculation of the neutron transmission through the powder sample. The resulting average decrease of the transmission due to the sulfur matrix is less than $0.13 \%$. 


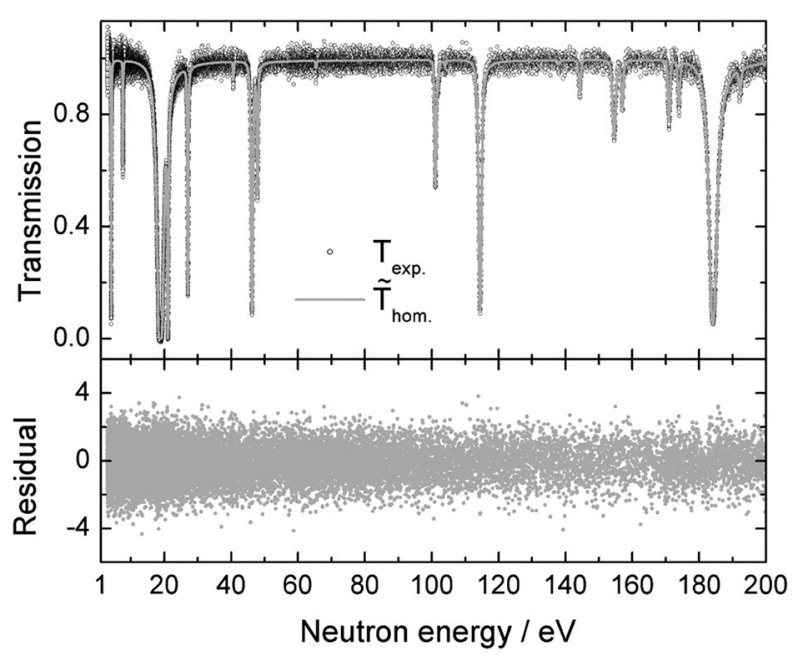

Fig. 4. Experimental transmission spectrum $T_{\exp }$. through the homogeneous metallic reference sample as function of neutron energy in the energy regions $3-200 \mathrm{eV}$ together with the calculated transmission spectrum $\tilde{T}_{\text {hom. }}$.
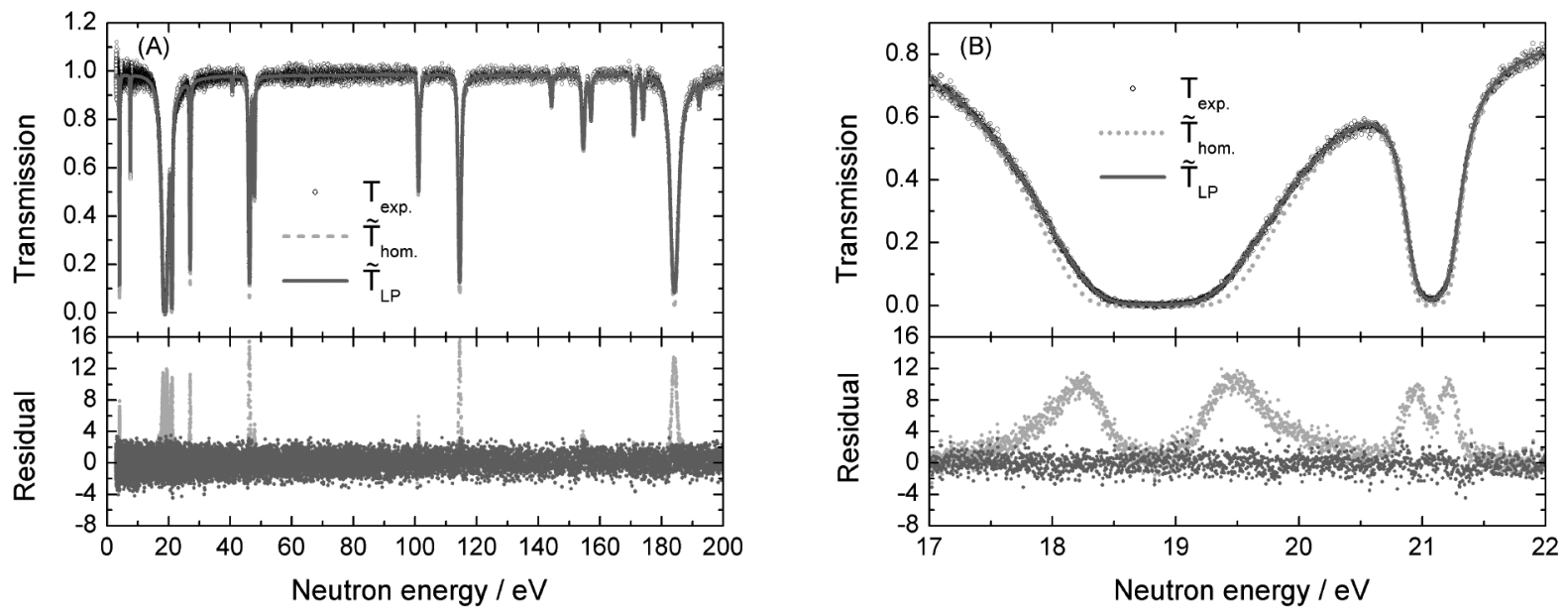

Fig. 5. Experimental transmission spectrum $T_{\exp }$. as function of neutron energy together with the calculated transmission spectra based on a homogeneous sample assumption $\left(\tilde{T}_{\text {hom. }}\right)$ or on the LP model $\left(\tilde{T}_{\mathrm{LP}}\right)$ using REFIT for the energy regions 3-200 eV (A) and 17-22 eV (B).

As mentioned in sect. 3, the areal density of the tungsten content could not be accurately deduced from the tungsten mass and sample area due to an inhomogeneous filling of the sample container. The transmission spectrum close to the weak resonances of ${ }^{183} \mathrm{~W}$ at $40.71 \mathrm{eV}, 144.33 \mathrm{eV}$ and $192.22 \mathrm{eV}$ was therefore used to determine the areal density by a fit to the data. For these resonances the particle self-shielding can be assumed to be small and the homogeneous assumption is valid (see sect. 4.2). The deduced density is $n_{W}=(1.084 \pm 0.014) \times 10^{-3}$ at $/ \mathrm{b}$.

Figure 5 compares the experimental and calculated transmission spectra in an overall energy range of 3-200 eV and in an extract of $17-22 \mathrm{eV}$. Applying the homogeneous sample assumption strong deviations between experimental and calculated transmission are observed in the region of strong resonances. For example, for the resonance of ${ }^{182} \mathrm{~W}$ resonance at $21.08 \mathrm{eV}$, the homogeneous model predicts a zero transmission. However, the experimental transmission reveals that due to the grain characteristics neutrons can still transverse the sample. In addition, the wing shape of the calculated transmission dip deviates significantly from the measured transmission dip, as indicated by the residuals. The maximum value of the residuals is 12 which means that this bias is not related to the uncorrelated uncertainty of the data. The bias is also not related to the uncertainty of the background model which results in a transmission uncertainty of less than 0.003 for the resonance dip. A similar behavior can be noticed for the ${ }^{186} \mathrm{~W}$ resonance at $18.83 \mathrm{eV}$. Finally, the $\chi^{2} / \nu$ was 4.7 .

Adjusting the areal density by a fit to the experimental transmission spectrum in the entire energy range from $3-200 \mathrm{eV}$ results in an areal density of $n_{W}=(0.939 \pm 0.001) \times 10^{-3}$ at $/ \mathrm{b}$. This result is not consistent with the density deduced from a fit using only weak resonances. Even though the $\chi^{2} / \nu$ improves to 2.5 the residual is still not flat. 
Table 1. Deduced tungsten areal densities from a fit using small resonances and from a fit using the entire spectrum and applying the LP model.

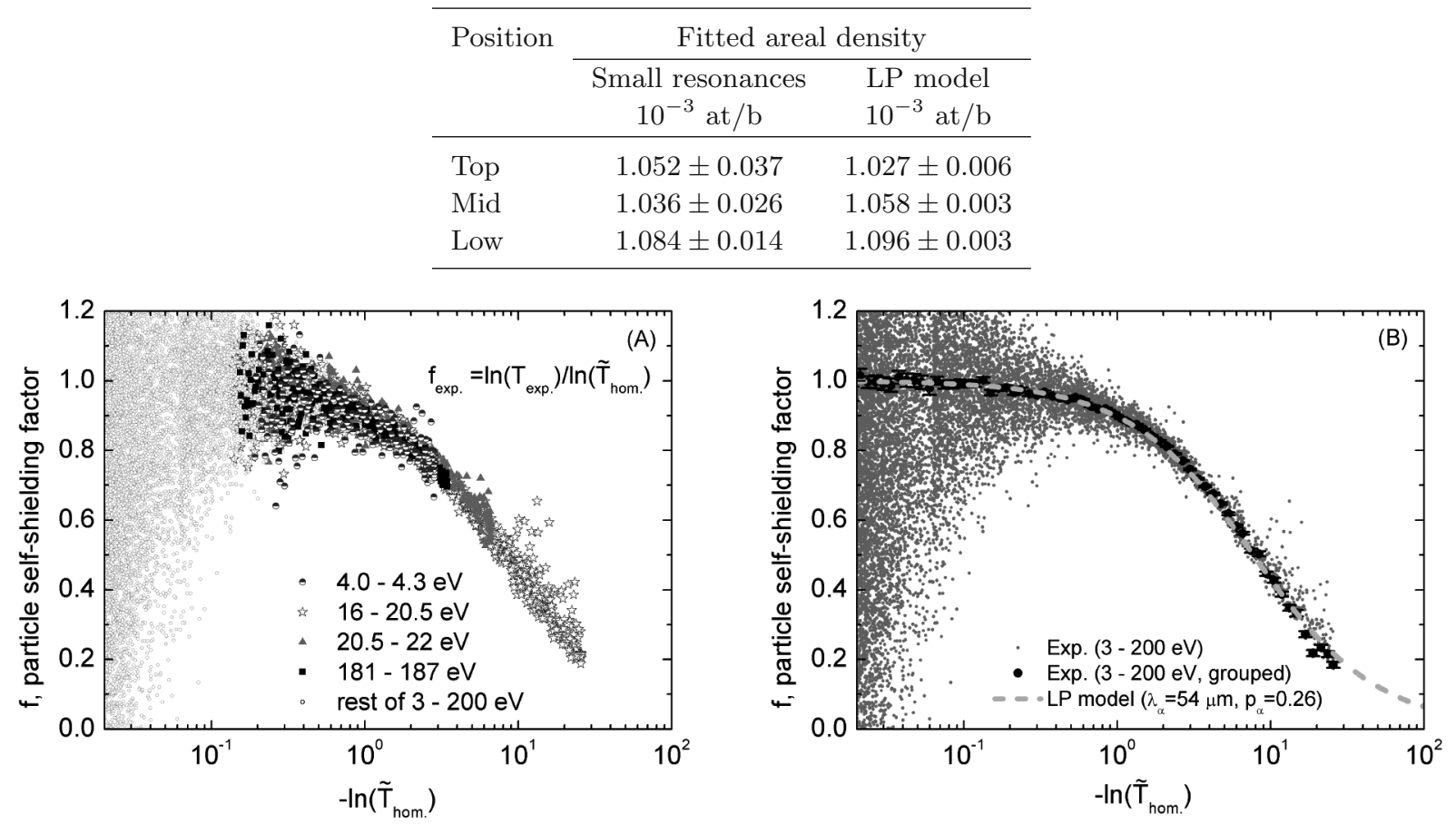

Fig. 6. (A) Experimental particle self-shielding factor as function of $-\ln \left(\tilde{T}_{\text {hom. }}\right)$ for different energy regions. (B) Experimental and calculated particle self-shielding factor as function of $-\ln \left(\tilde{T}_{\text {hom. }}\right)$.

The LP model was applied in a second calculation of the transmission spectrum shown in fig. 5. In the calculation, the two model parameters $\lambda_{\alpha}$ and $p_{\alpha}$ together with the tungsten areal density were adjusted in a fit to the experimental data. Component $\beta$ was assumed to consist of a homogeneous mixture of the void between particles and the sulfur particles using the average sulfur content. The quality of the fit improved significantly compared to using the homogeneous model. This can be noticed from the flat residuals close to the strong resonances shown in fig. 5 and from the $\chi^{2} / \nu$ which decreased to 1.0. The model parameters $\lambda_{\alpha}$ and $p_{\alpha}$ were deduced to be $54 \pm 10 \mu \mathrm{m}$ and $26 \pm 3 \%$, respectively. The obtained areal density $n_{W}=(1.096 \pm 0.003) \times 10^{-3}$ at/b using the LP model is consistent with the result deduced from a fit using only weak resonances. The uncertainties of the fitted parameters $\boldsymbol{\theta}$ were determined by conventional uncertainty propagation using [29]

$$
V_{\boldsymbol{\theta}}=\left(G_{\boldsymbol{\theta}}^{T} V_{\boldsymbol{T}}^{-1} G_{\boldsymbol{\theta}}\right)^{-1}
$$

where $V_{\boldsymbol{\theta}}$ and $V_{\boldsymbol{T}}$ are the parameter and experimental covariances. $G_{\boldsymbol{\theta}}$ is the sensitivity matrix of the transmission with respect to the fitted parameters. The experimental covariance matrix included the uncorrelated uncertainty component due to counting statistics as well as the correlated components due to the normalization $N$, the background $k$ and the thickness of the pressed powder layer. The latter has no impact on the uncertainty of the fitted areal density. Note that REFIT uses only the uncorrelated uncertainty in the $\chi^{2}$ minimization.

The consistency between deducing the tungsten areal density from a fit using small resonances and from a fit using the entire spectrum and applying the LP model was checked by performing two additional measurements. For theses measurements the sample was positioned either with the center or with the upper part in the neutron beam. Table 1 summarizes the results of the areal density adjustments. Both methods are consistent within the quoted uncertainties.

\subsection{Particle self-shielding factor}

The particle self-shielding factor $f$ can be estimated using directly the experimental transmission spectrum,

$$
f_{\text {exp } .}=\frac{\ln \left(T_{\text {exp } .}\right)}{\ln \left(\tilde{T}_{\text {hom. }}\right)},
$$

where $\tilde{T}_{\text {hom. }}$ is the transmission through a homogeneous sample, calculated by REFIT accounting for Doppler broadening and the response of the TOF-spectrometer. Figure 6(A) shows the experimental particle self-shielding factor as 
function of $-\ln \left(\tilde{T}_{\text {hom. }}\right)$ using the transmission data in different energy regions. The factor decreases slowly with decreasing transmission up to $-\ln \left(\tilde{T}_{\text {hom. }}\right)=0.1$. For a transmission larger than $\tilde{T}_{\text {hom. }}=0.85$, the particle self-shielding factor deviates only by $2 \%$ from unity which means that the impact of the heterogeneous characteristics of the sample becomes small. This was the motivation to use weak resonance for the determination of the areal density. Above $-\ln \left(\tilde{T}_{\text {hom. }}\right)=$ 0.1, the factor drops rapidly. The strongest self-shielding occurs for the ${ }^{186} \mathrm{~W}$ resonance at $18.83 \mathrm{eV}$. By using only this single resonance a decrease in particle self-shielding factor from about 0.9 to 0.2 can directly be measured.

In fig. 6(B) the experimental particle self-shielding factor is based on all data points between $3-200 \mathrm{eV}$. The factor is also given as grouped values with 20 values per decade. For comparison, a calculation of the particle self-shielding factor based on LP model (eq. (9)) is shown as well. In the calculation the model parameters, $\lambda_{\alpha}=54 \mu \mathrm{m}$ and $p_{\alpha}=26 \%$, were used which were deduced in sect. 4.1 from a fit to the transmission spectrum. It can be noticed that the experimental particle self-shielding factor has the same characteristic shape as the theoretical one. In particular the region where the factor drops rapidly is well reproduced.

\section{Conclusion}

In this contribution the particle self-shielding of tungsten grains in a powder sample has been investigated using neutron resonance transmission analysis. The shortcoming of a homogeneous sample assumption has been demonstrated based on measured transmission spectra. Applying the stochastic model of Levermore et al. [15] and fitting the effective characteristic length and volume fraction of the tungsten grains, an agreement between calculation and experiment was obtained.

An approximate particle self-shielding factor was directly deduced from the experimental results. The factor shows a similar cross section dependent behavior as the one calculated using the Levermore-Pomraning model.

We are grateful for the support by the GELINA technical staff and by the EC-JRC-IRMM sample preparation group. This work was partly supported by the European Commission through the project ERINDA (FP7-269499) and is part of a collaboration between EC-JRC-IRMM and JAEA. We also acknowledge the work of the anonymous referees for their valuable comments and suggestions which improved the original manuscript.

Open Access This is an open access article distributed under the terms of the Creative Commons Attribution License (http://creativecommons.org/licenses/by/4.0), which permits unrestricted use, distribution, and reproduction in any medium, provided the original work is properly cited.

\section{References}

1. W.B. Doub, Nucl. Sci. Eng. 10, 299 (1961).

2. P. Schillebeeckx, B. Becker, Y. Danon, K. Guber, H. Harada, J. Heyse, A.R. Junghans, S. Kopecky, C. Massimi, M. Moxon, N. Otuka, I. Sirakov, K. Volev, Nucl. Data Sheets 113, 3054 (2012) DOI: 10.1016/j.nds.2012.11.005.

3. P. Schillebeeckx, A. Borella, F. Emiliani, G. Gorini, W. Kockelmann, S. Kopecky, C. Lampoudis, M. Moxon, E. Perelli Cippo, H. Postma, N.J. Rhodes, E.M. Schooneveld, C. Van Beveren, JINST 7, C03009 (2012) DOI: 10.1088/17480221/7/03/C03009.

4. C.H. Randall, Naval Reactor Physics Book, edited by A. Radkowsky, Vol. 1 (Naval Reactors, Division of Reactor Development, United States Atomic Energy Commission, 1964).

5. M.M.R. Williams, Random Processes in Nuclear Reactors (Pergamon Press, 1974).

6. R. Sanchez, G.C. Pomraning, Ann. Nucl. Energy 18, 371 (1991) DOI: 10.1016/0306-4549(91)90073-7.

7. G.A. Titov, J. Atmos. Sci. 47, 24 (1990) DOI: 10.1175/1520-0469(1990)047<0024:SDORTI>2.0.CO;2.

8. F. Malvagi, R.N. Byrne, G.C. Pomraning, R.C.J. Somerville, J. Atmos. Sci. 50, 2146 (1993) DOI: 10.1175/15200469(1993)050<2146:SRTIPC $>2.0 . \mathrm{CO} ; 2$.

9. A. Marshak, A.B. Davis (Editors), 3D Radiative Transfer in Cloudy Atmosphere, Vol. XII (Springer, Heidelberg, Germany, 2005).

10. P. Boissé, Astron. Astrophys. 228, 483 (1990).

11. M.P. Hobson, R. Padman, Mon. Not. R. Astron. Soc. 264, 161 (1993).

12. K.M. Case, F. De Hoffman, G. Placzek, Introduction to the Theory of Neutron Diffusion, Vol. 1 (Los Alamos Scientific Laboratory, 1953).

13. H. Hurwitz, P.F. Zweifel, Nucl. Sci. Eng. 1, 438 (1956).

14. W.R. Burrus, Radiation transmission through boral and similar heterogeneous materials consisting of randomly distributed absorbing chunks, Report ORNL-2528, Oak Ridge National Laboratory (1960).

15. C.D. Levermore, G.C. Pomraning, D.L. Sanzo, J. Wong, J. Math. Phys. 27, 2526 (1986) DOI: 10.1063/1.527320.

16. D. Vanderhaegen, J. Quant. Spectrosc. Radiat. Transfer 36, 557 (1986) DOI: 10.1016/0022-4073(86)90128-7. 
17. M.M.R. Williams, Ann. Nucl. Energy 35, 750 (2008) DOI: 10.1016/j.anucene.2007.08.017.

18. P.A. Rosen, J.M. Foster, M.J. Taylor, P.A. Keiter, C.C. Smith, J.R. Finke, M. Gunderson, T.S. Perry, Astrophys. Space Sci. 307, 213 (2007) DOI: 10.1007/s10509-006-9235-4.

19. P. Keiter, M. Gunderson, J. Foster, P. Rosen, A. Comley, M. Taylor, T. Perry, Phys. Plasmas 15, 056901 (2008) DOI: $10.1063 / 1.2927529$.

20. E. Kassianov, D. Veron, J. Quant. Spectrosc. Radiat. Transfer 122, 566 (2011) DOI: 10.1016/j.jqsrt.2010.06.011.

21. M.C. Moxon, J.B. Brisland, GEEL REFIT, A least squares fitting program for resonance analysis of neutron transmission and capture data computer code, AEA-InTec-0630, AEA Technology (October, 1991).

22. B. Becker, K. Kauwenberghs, S. Kopecky, H. Harada, M. Moxon, P. Schillebeeckx, JRC Scientific and Policy Report 86936, ISBN 978-92-79-35095-5, ISSN 1831-9424, DOI: 10.2787/84664 (2013).

23. W. Mondelaers, P. Schillebeeckx, Not. Neutroni Luce Sincrotrone 11, 19 (2006).

24. J. Gonzalez, C. Bastian, S. de Jonge, K. Hofmans, Modular Multi-Parameter Multiplexer MMPM, Hardware description and user guide, Internal Report GE/R/INF/06/97, IRMM, Geel.

25. A. Moens, G. Sibbens, private communication (2013).

26. D. Ene, C. Borcea, S. Kopecky, W. Mondelaers, A. Negret, A.J.M. Plompen, Nucl. Instrum. Methods A 618, 54 (2010) DOI: $10.1016 /$ j.nima.2010.03.005.

27. B. Becker, C. Bastian, F. Emiliani, F. Gunsing, J. Heyse, K. Kauwenberghs, S. Kopecky, C. Lampoudis, C. Massimi, N. Otuka, P. Schillebeeckx, I. Sirakov, JINST 7, P11002 (2012) DOI: 10.1088/1748-0221/7/11/P11002.

28. F. Emiliani, B. Becker, K. Guber, S. Kopecky, P. Schillebeeckx, R. Wynants, JRC Scientific and Policy Report 88008, ISBN 978-92-79-35264-5, ISSN: 1831-9424, DOI: 10.2787/86701 (2013).

29. I. Lira, Evaluating the measurement uncertainty (IOP Publishing Ltd., Bristol and Philadelphia, 2002) ISBN 0-7503-0840-0. 